\title{
Association of IFN- $\gamma$ : IL-10 Cytokine Ratio with Nonsegmental Vitiligo Pathogenesis
}

\author{
Yaswanth Ala, ${ }^{1}$ Mohammed Khalid Pasha, ${ }^{1}$ Raja Narasimha Rao, \\ Prasanna Latha Komaravalli, ${ }^{1}$ and Parveen Jahan ${ }^{1,3}$ \\ ${ }^{1}$ Department of Genetics, Osmania University, Hyderabad, Telangana 500007, India \\ ${ }^{2}$ Central Railway Hospital, Lallaguda, Hyderabad, Telangana 500017, India \\ ${ }^{3}$ Department of Zoology, School of Sciences, Maulana Azad National Urdu University, Gachibowli, Hyderabad, \\ Telangana 500032, India
}

Correspondence should be addressed to Parveen Jahan; dr.pjahan@gmail.com

Received 20 June 2015; Revised 20 August 2015; Accepted 20 August 2015

Academic Editor: Xu-Jie Zhou

Copyright (C) 2015 Yaswanth Ala et al. This is an open access article distributed under the Creative Commons Attribution License, which permits unrestricted use, distribution, and reproduction in any medium, provided the original work is properly cited.

\begin{abstract}
Background and Objectives. Cytokines regulate immune response and inflammation and play a crucial role in depigmentation process of vitiligo. The present study aimed to estimate the serum levels of pro- and anti-inflammatory cytokines, IFN- $\gamma$ and IL10 , and their ratios in nonsegmental vitiligo patients and healthy individuals from India. Methods. Blood samples were collected from 280 subjects and serum IFN- $\gamma$ and IL-10 levels were measured using standard ELISA. Results. Nonsegmental vitiligo patients showed increased levels of IFN- $\gamma(12.4 \pm 3.2$ versus $9.9 \pm 4.4 \mathrm{pg} / \mathrm{mL})$ and decreased levels of IL-10 $(9.3 \pm 1.7 \mathrm{versus} 11.5 \pm 5 \mathrm{pg} / \mathrm{mL})$ compared to controls. Ratio of IFN- $\gamma$ : IL-10 differed significantly from patients to controls $(p<0.05)$. IFN- $\gamma$ concentrations and IFN- $\gamma$ : IL-10 ratio varied significantly with respect to clinical variants, disease stability, and social habits (smoking and alcohol consumption) and showed a positive correlation with disease duration. Family history of vitiligo was significantly associated with IFN- $\gamma$ : IL-10 ratio but not with their individual levels. Conclusion. The ratio of IFN- $\gamma$ : IL-10 serum levels may be considered as one of the promising immunological markers in nonsegmental vitiligo. This is the first study considering multiple aspects in relation to ratio of cytokine levels. Similar studies with large samples are warranted to confirm our observations.
\end{abstract}

\section{Introduction}

Vitiligo is defined as an acquired cutaneous complex disorder resulting from functional loss of melanocytes in epidermis, characterized by milky white macules of various sizes and shapes that tend to enlarge peripherally in the course of time [1]. It is an inflammatory disorder associated with increased expression of inflammatory cytokines in the skin and blood [2]. The melanocytes are targeted by multiple aggressions leading to marked reduction and destruction of pigment cells in vitiligo patients [3]. The prevalence of the disease is estimated to be $<0.1 \%$ to $>8 \%$ of the world population and is found to be $0.5-2.5 \%$ in India with a high prevalence of $8.8 \%$ in Gujarat and Rajasthan states $[4,5]$. The pathogenesis of vitiligo includes both intrinsic defects within melanocytes that activate cellular stress response and the autoimmune mechanisms targeting the melanocytes involving both humoral and cell mediated immunity [57]. Infiltration of cytotoxic $T$ cells in perilesional lesions is the characteristic hallmark of vitiligo [8]. There is growing evidence that cytokines are important in the depigmentation process and show a cytokine imbalance in the skin of vitiligo patients suggesting their prominent role in autoimmune pathogenesis $[9,10]$. Systemic biological therapies used for treating psoriasis and other autoimmune diseases by targeting cytokines indicate that a similar approach might be effective for vitiligo [6].

Cytokines are protein molecules that include Interferons (IFNs), Interleukins (ILs), various Colony Stimulating Factors (CSFs), and Tumor Necrosis Factors (TNFs) which are key molecules in mediating inflammatory and cytokine reactions. Their response due to imbalance or deficiency in 
the cytokine network may largely determine autoimmune disease susceptibility and severity. Alteration in the concentration of various proinflammatory and anti-inflammatory cytokines such as IL-6, IL-8, IL-10, IL-2, TNF- $\alpha$, and IFN- $\gamma$ has been associated with various autoimmune disorders [1113]. IL-10 is a potent regulator of anti-inflammatory immune responses and is hence considered to be a downregulator of cytokine production by Thl cells and macrophages [14, 15]. On the other hand, elevated concentrations of IFN- $\gamma$ initiate apoptosis and it has been suggested that melanocyte death is mediated by apoptosis in the context of autoimmunity [16]. In view of the above literature, we aimed to assess the balance between the proinflammatory IFN $-\gamma$ and anti-inflammatory IL-10 cytokine serum levels in NSV patients and healthy controls from India.

\section{Materials and Methodology}

The present study enrolled a total of 280 subjects that included 130 patients (mean age $27.6 \pm 6.9$ years) and 150 healthy controls (mean age $26.9 \pm 5.6$ years) from Central Railway Hospital, Hyderabad, India, in the period of April 2012 to March 2014. Approval for the study was obtained from the Institutional Ethical Committee, Osmania University, Hyderabad, India. The subjects were enrolled after providing informed consent and detailed information was procured from all the subjects regarding demographic, clinical, and family history of vitiligo. The patients enrolled were either on no drug treatment or in the washout period of three months.

Information was recorded in a pro forma with respect to clinical presentation of the disease, age, gender, age at onset, family history, dietary habits, occupation, socioeconomic background, associated diseases, and clinical parameters. Five millilitres $(\mathrm{mL})$ of blood sample was collected from each of the enrolled patients and controls. An inclusion criterion of patients was presence of nonsegmental vitiligo without any other associated disorder as well as not being under any treatment. Patients with segmental vitiligo, known allergies, other skin diseases, or other autoimmune disorders such as Hashimoto's thyroiditis, Graves' disease, type 1 insulindependent diabetes mellitus, Addison's disease, psoriasis, rheumatoid arthritis, and thyroid dysfunction which are known to have altered levels of cytokines were excluded from the study $[11,17]$.

Based upon the spreading of the lesions or white macules at the time of sample collection, the patients were categorized into active vitiligo (existing lesions are spreading and/or new lesions had appeared within the previous six months) and stable vitiligo (no increase in the size or number of lesions within 6 months or more) [18]. The duration of the disease was considered as the time between initial appearance of the clinical symptoms of the disease and sample collection.

2.1. Determination of Serum IL-10 and IFN- $\gamma$ Levels. Analysis was performed on blood serum using sandwich ELISA method (eBioscience Inc., San Diego, CA, USA). In brief, the antibody was coated onto a 96-well plate by adding $100 \mu \mathrm{L}$ of capture antibody (1X) to each well and incubated overnight at $4^{\circ} \mathrm{C}$. The wells were blocked with $200 \mu \mathrm{L} /$ well of
1X assay diluent and incubated for 1 hour at room temperature (RT). After that, $100 \mu \mathrm{L} /$ well of serum samples and standards was added to the wells and incubated for 2 hours at RT. After incubation, detection antibody $(100 \mu \mathrm{L} /$ well $)$ was added and incubated for 1 hour. Then $100 \mu \mathrm{L} /$ well of avidin-HRP (Horseradish Peroxidase) was added and incubated at RT for another 30 minutes. The plate was incubated with substrate (100 $\mu \mathrm{L} /$ well) for 15 minutes at RT followed by $(50 \mu \mathrm{L} /$ well) stop solution. Each of the above steps was interspersed by 5-7 washes to ensure no carryover and absorbance was measured at $450 \mathrm{~nm}$ and $540 \mathrm{~nm}$. The concentrations were calculated using MPM software version 6.1.

2.2. Statistical Analysis. Data was analysed using descriptive statistics to calculate the percentages, mean values, and standard deviation. Student's " $t$-" test, one-way ANOVA, and Pearson correlation were carried out to analyse the variation between patients and controls, clinical variants of vitiligo, and association among the variables, respectively. A $p$ value less than 0.05 was considered as statistically significant.

\section{Results}

A total of 130 vitiligo patients with a mean age of $27.6 \pm 6.9$ years and 150 healthy controls with a mean age of $26.9 \pm$ 5.6 years were enrolled in this study. Approximately, equal numbers of males and females were observed in both of the study groups. The mean age at onset of the disease was $23.3 \pm 7.0$ years $(23.2 \pm 7.8$ years in females and $23.4 \pm 6.5$ years in males) and the duration of the disease ranged from 1 to 14 years with a mean of $4.2 \pm 3.1$ years $(4.6 \pm 3.5$ years in females and $3.8 \pm 2.8$ years in males). In the present study $63.8 \%$ of the patients showed active vitiligo and $36.2 \%$ stable vitiligo; family history of NSV was seen in $40 \%$ of patients and $32 \%$ of male patients had the social habits of smoking and alcohol consumption (Table 1).

3.1. IFN- $\gamma$ Serum Concentrations. The proinflammatory cytokine IFN- $\gamma$ levels were noted to be significantly elevated in vitiligo patients compared to healthy controls (12.4 \pm $3.2 \mathrm{pg} / \mathrm{mL}$ versus $9.9 \pm 4.4 \mathrm{pg} / \mathrm{mL} ; p<0.05)$. Analysis of variance (ANOVA) showed a significant difference with respect to serum IFN- $\gamma$ levels among the clinical variants of vitiligo (nondermatomal, acrofacial, mucosal, and focal) $(p<0.05)$. Increased amounts of IFN- $\gamma$ were observed in active vitiligo patients compared to patients with the stable condition of the disease $(p<0.05)$. With respect to social habits (smoking and alcohol consumption), the cytokine levels were analysed for only male patients and controls as none of our female subjects were with these social habits.

Significantly elevated concentrations of IFN- $\gamma$ were noticed in patients compared to controls $(p=0.001)$ and in patients with social habits compared to patients without social habits $(p=0.04)$. However, the levels were not significant between controls with and without social habits $(p=0.156)$. IFN- $\gamma$ levels were nearly similar in patients with and without family history of vitiligo $(p>0.05)$ (Table 2$)$ (Figure 1). A positive correlation was observed between the 
TABLE 1: Demographic and clinical characteristics of subjects included in the study.

\begin{tabular}{|c|c|c|c|}
\hline & $\begin{array}{c}\text { Patients }(n=130) \\
\text { Mean } \pm \text { SD }\end{array}$ & $\begin{array}{c}\text { Controls }(n=150) \\
\text { Mean } \pm \text { SD }\end{array}$ & $p$ value \\
\hline Age in years & $27.6 \pm 6.9$ years & $26.9 \pm 5.6$ & 0.34 (NS) \\
\hline Males & $27.3 \pm 6.7(76)$ & $27.2 \pm 6.0(85)$ & $0.92(\mathrm{NS})$ \\
\hline Females & $27.9 \pm 7.3(54)$ & $26.3 \pm 4.7(65)$ & 0.15 (NS) \\
\hline Duration of the disease & $4.2 \pm 3.1$ years & NA & \\
\hline Males & $3.8 \pm 2.8$ years & & \\
\hline Females & $4.6 \pm 3.5$ years & & \\
\hline Age at onset & $23.3 \pm 7.0$ years & NA & \\
\hline Males & $23.4 \pm 6.5$ years & & \\
\hline \multirow[t]{2}{*}{ Females } & $23.2 \pm 7.8$ years & & \\
\hline & $n(\%)$ & $n(\%)$ & \\
\hline \multicolumn{4}{|l|}{ Males with social habits } \\
\hline Yes & $29(22.3)$ & $20(23.5)$ & \\
\hline No & $47(77.7)$ & $65(76.5)$ & \\
\hline \multicolumn{4}{|c|}{ Clinical variants of vitiligo } \\
\hline Nondermatomal & $58(44.6)$ & NA & \\
\hline Acrofacial & $33(25.4)$ & - & \\
\hline Mucosal & $22(17)$ & - & \\
\hline Focal & $17(13)$ & - & \\
\hline \multicolumn{4}{|l|}{ Disease status } \\
\hline Stable & $47(36.2)$ & NA & \\
\hline Active & $83(63.8)$ & - & \\
\hline \multicolumn{4}{|l|}{ Family history } \\
\hline Yes & $52(40)$ & & \\
\hline No & $78(60)$ & - & \\
\hline
\end{tabular}

Note: NS: not significant; $n=$ number of subjects, and SD = standard deviation.

concentration of this proinflammatory cytokine and the duration of the disease (Figure 2).

3.2. IL-10 Serum Concentrations. A significant difference was found in the mean serum concentrations of the antiinflammatory cytokine IL-10 between patients and controls $(9.3 \pm 1.7 \mathrm{pg} / \mathrm{mL}$ versus $11.5 \pm 5 \mathrm{pg} / \mathrm{mL} ; p<0.05)$. However, no difference in the levels of IL-10 with respect to clinical variants, disease stability, social habits, and family history within the patient group was seen $(p>0.05)$ (Table 2 , Figure 1). Furthermore, we did not find correlation between the duration of disease and serum levels of IL-10 ( $p>0.05)$ (Figure 2).

A positive correlation between the concentrations of the two cytokines IL-10 and IFN- $\gamma$ was seen in patients but not in controls. Similarly, active vitiligo patients exhibited a positive correlation between IL-10 and IFN- $\gamma$ but the stable vitiligo patients did not (Figure 2).

3.3. Ratio of IFN- $\gamma: I L-10$ in Serum. The ratio of IFN- $\gamma$ to IL-10 was detected to be significantly higher in NSV patients compared to controls $(1.3 \pm 0.3$ versus $0.9 \pm 0.7$; $p<0.05)$. Patients with clinical variants, with active and stable vitiligo, with and without social habits of smoking and alcohol consumption, and with and without family history of nonsegmental vitiligo also exhibited a significantly increased ratio of IFN- $\gamma$ : IL-10 ( $p<0.05)$ (Table 2, Figure 1). Further, there was a positive correlation between this ratio and the duration of the disease $(p<0.05)$ (Figure 2).

\section{Discussion}

In the present study, the serum concentration of the proinflammatory cytokine IFN- $\gamma$ was significantly elevated in NSV patients compared to controls. Supporting our observation, Th1 predominance has been reported to be associated with vitiligo from previous studies. Shi and Erf in Smyth line (SL) chicken model for autoimmune vitiligo have shown that IFN- $\gamma$, a proinflammatory cytokine which acts as a signature cytokine of Th1 mediated immunity, has remarkably increased in the Smyth line vitiligo samples proving the central role of this cytokine in the SLV pathomechanism [19]. Rashigi et al. reported that both vitiligo patients and mouse model of vitiligo reflect a uniquely IFN- $\gamma$ specific Th1 cytokine signature in the skin that includes IFN- $\gamma$ dependent chemokines such as CXCL9, CXCL10, and CXCL11 which induces $\mathrm{T}$ cell homing into peripheral tissues [6]. Mechanistic studies in mouse model revealed that depigmentation 
TABLE 2: Serum concentrations of IFN- $\gamma$, IL-10, and IFN- $\gamma$ : IL-10 ratio in controls and vitiligo patients.

\begin{tabular}{|c|c|c|c|}
\hline & $\begin{array}{c}\text { IFN }-\gamma \text { in } \\
\mathrm{pg} / \mathrm{mL} \\
\text { Mean } \pm \mathrm{SD}\end{array}$ & $\begin{array}{c}\text { IL-10 } \\
\text { in pg/mL } \\
\text { Mean } \pm \text { SD }\end{array}$ & $\begin{array}{c}\text { IFN- } \gamma: \text { IL-10 } \\
\text { in pg/mL } \\
\text { Mean } \pm \text { SD }\end{array}$ \\
\hline Vitiligo patients (130) & $12.4 \pm 3.2$ & $9.3 \pm 1.7$ & $1.3 \pm 0.3$ \\
\hline Controls (150) & $9.9 \pm 4.4$ & $11.5 \pm 5.0$ & $0.9 \pm 0.7$ \\
\hline " $t$-" test $p$ value & $0.0001^{*}$ & $0.0001^{*}$ & $0.0001^{*}$ \\
\hline \multicolumn{4}{|l|}{ Clinical variants of vitiligo (\%) } \\
\hline Nondermatomal (44.6) & $12.5 \pm 3.2$ & $9.7 \pm 1.8$ & $1.2 \pm 0.2$ \\
\hline Acrofacial (25.4) & $14.4 \pm 2.7$ & $8.9 \pm 1.2$ & $1.6 \pm 0.3$ \\
\hline Mucosal (17) & $10.5 \pm 3.1$ & $9.1 \pm 2.1$ & $1.1 \pm 0.3$ \\
\hline Focal (13) & $11.0 \pm 2.0$ & $8.9 \pm 1.7$ & $1.2 \pm 0.1$ \\
\hline One-way ANOVA “p” value & $0.000^{*}$ & $0.111(\mathrm{NS})$ & $0.000^{*}$ \\
\hline \multicolumn{4}{|l|}{ Disease status (\%) } \\
\hline Stable (36.2) & $11.5 \pm 2.7$ & $9.3 \pm 1.8$ & $1.2 \pm 0.3$ \\
\hline Active (63.8) & $13.0 \pm 3.3$ & $9.3 \pm 1.7$ & $1.4 \pm 0.3$ \\
\hline " $t$-" test $p$ value & $0.009^{*}$ & $1(\mathrm{NS})$ & $0.0004^{*}$ \\
\hline \multicolumn{4}{|l|}{ Family history (\%) } \\
\hline Yes (40) & $12.9 \pm 3.4$ & $9.1 \pm 1.6$ & $1.4 \pm 0.3$ \\
\hline No $(60)$ & $12.1 \pm 3.1$ & $9.4 \pm 1.8$ & $1.3 \pm 0.3$ \\
\hline " $t$-" test $p$ value & 0.1 (NS) & 0.3 (NS) & $0.02^{*}$ \\
\hline \multicolumn{4}{|l|}{ Male patients with SH (\%) } \\
\hline Yes (42) & $14.2 \pm 4.1$ & $9.9 \pm 2.0$ & $1.45 \pm 0.3$ \\
\hline No (58) & $10.9 \pm 2.8$ & $9.1 \pm 2.0$ & $1.2 \pm 0.3$ \\
\hline " $t$-" test $p$ value & $0.0004^{*}$ & 0.09 (NS) & $0.0008^{*}$ \\
\hline
\end{tabular}

Note: NS: not significant; ${ }^{*} p<0.05, n=$ number of subjects, $\mathrm{SD}=$ standard deviation, and $\mathrm{SH}=$ social habits.

requires IFN- $\gamma$, which induces the local accumulation of melanocyte specific $\mathrm{CD} 8^{+} \mathrm{T}$ cells within the skin supporting the critical role of IFN- $\gamma$ in vitiligo [20]. Further, Nigam et al. also reported an increased number of $\mathrm{CD} 8^{+} \mathrm{T}$ cells in patients with vitiligo compared to controls [21]. Gregg et al. suggested that vitiligo is entirely dependent on $\mathrm{CD} 8^{+} \mathrm{T}$ cells, while $\mathrm{CD} 4^{+} \mathrm{T}$ cells exert a negative regulatory effect and the genetic ablation of IFN $-\gamma$ resulted in scarce $\mathrm{CD}^{+} \mathrm{T}$ cell infiltration into the skin [22]. Skin explant model studies reported that the stronger the $\mathrm{CD}^{+} \mathrm{T}$ cell response the more elaborate the vitiligo [8]. IFN- $\gamma$ indirectly increases the expression of intercellular adhesion molecule-1 (ICAM-1) on melanocytes and enhances $\mathrm{T}$ cell-melanocyte attachment in the skin and thus establishes a link between cytokine and $\mathrm{T}$ cell mediated destruction of melanocytes in vitiligo $[23,24]$.

Decreased serum concentrations of the anti-inflammatory cytokine IL-10 were observed in patients compared to controls suggesting low Th2 cytokine profile in the pathogenesis of vitiligo. Shi and Erf suggested that the physiological inducer of $\mathrm{T}$ regulatory cells (Tregs) function and proliferation, IL-10 cytokine, was found to be decreased in active vitiligo lesions [19]. Taher et al. and Tembhre et al. had reported increased levels of this immunosuppressive cytokine in vitiligo patients who showed the repigmentation process upon treatment with tacrolimus and narrow band ultraviolet B (NB-UVB), respectively [17, 25]. This indicates that upregulation of IL-10 may be responsible for the drug response which indirectly supports the decreased levels of IL10 in vitiligo pathogenesis observed in our study.

Contrary to the serum levels observed at systemic level in the present study, Grimes et al. reported increased IL-10 mRNA levels in involved and uninvolved tissue of vitiligo [26]. Estimation of the cytokines in the involved tissue and circulating levels together in the same patients and comparison with normal healthy controls may help in understanding the discrepancy in the results.

The higher IFN- $\gamma$ :IL-10 ratio noted in our patients compared to controls corroborates the cell based studies of Dwivedi et al. and Nigam et al. from India who observed a decrease in the ratio of $\mathrm{CD}^{+} / \mathrm{CD}^{+} \mathrm{T}$ cells in vitiligo patients compared to controls signifying the role of $\mathrm{T}$ cell mediated pathogenesis in vitiligo $[18,21]$. The impaired cytokine network might contribute to the reduction and loss of functional Tregs which are involved in immune tolerance [27]. This implies that the balance between pro- and antiinflammatory cytokines may play an important role in the pathogenesis of NSV.

Another significant observation of the present study was increase in the anti-inflammatory cytokine with an increase in the proinflammatory cytokine exhibiting a positive correlation between IL-10 concentrations and the IFN- $\gamma$ levels in NSV patients indicating that there might be a concomitant 

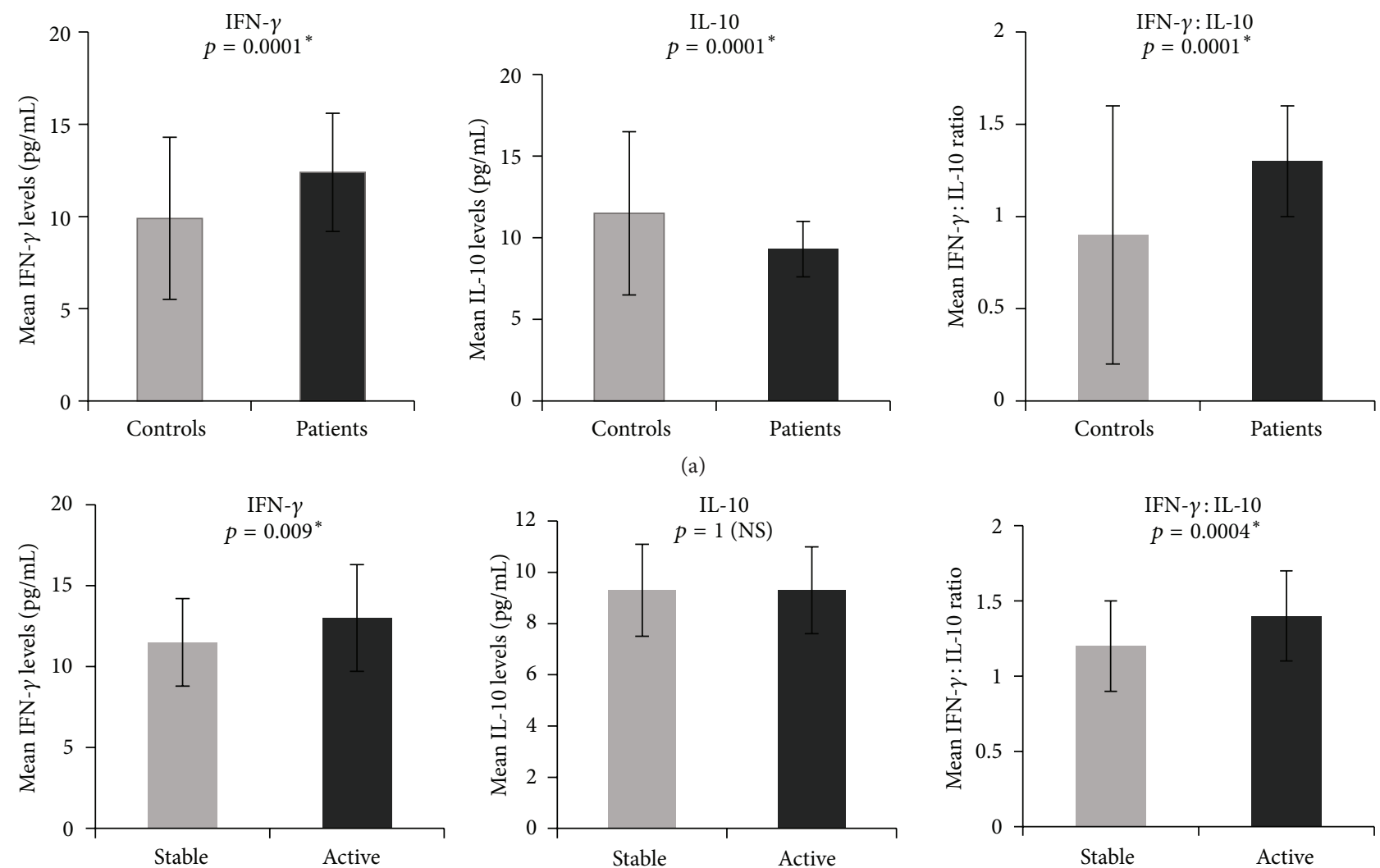

(a)
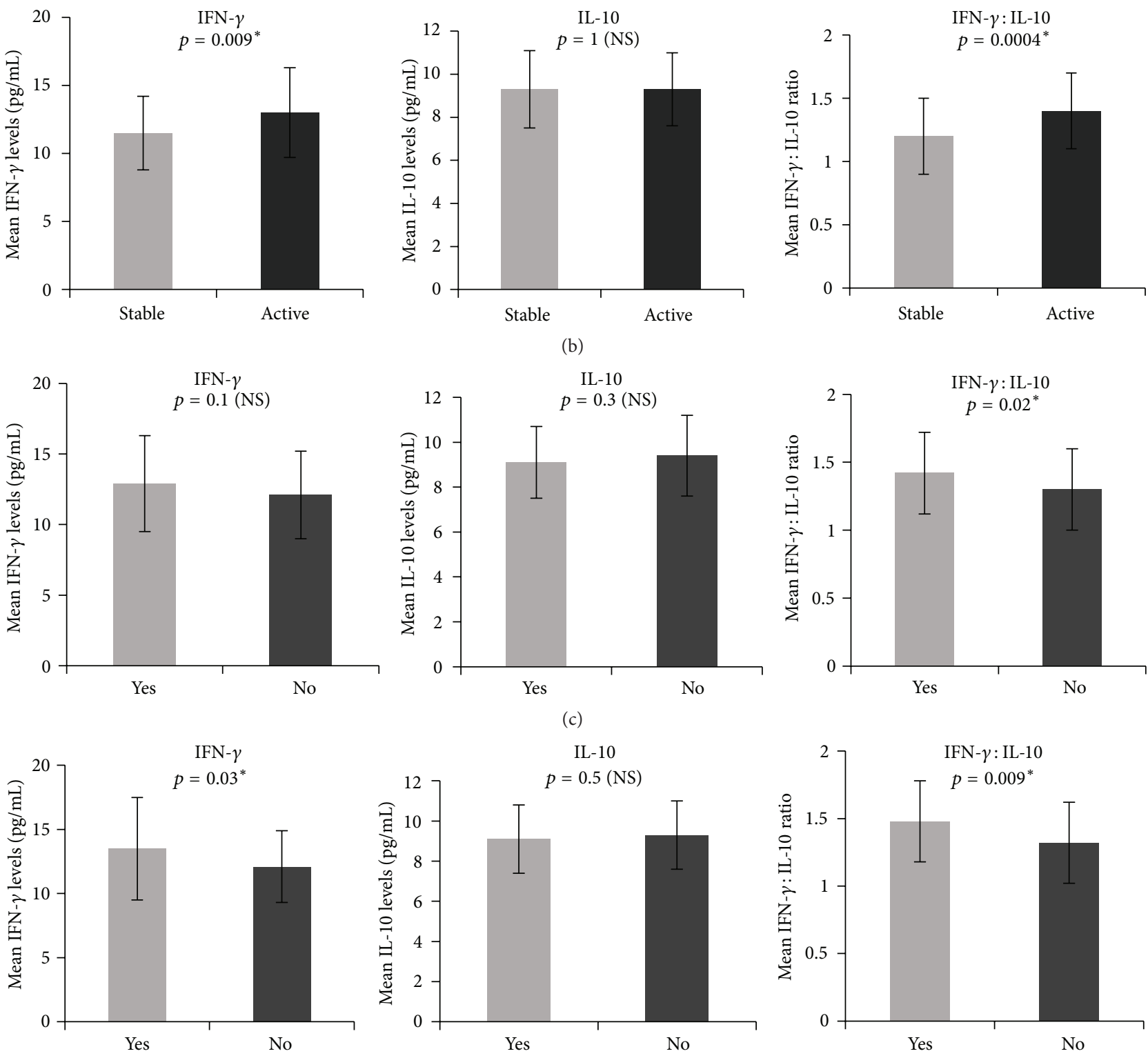

(d)

FIGURE 1: Representing mean IFN- $\gamma$, IL-10, and IFN- $\gamma$ : IL-10 ratio in (a) patients and controls, (b) patients with active and stable vitiligo, (c) patients with family history of vitiligo, and (d) patients with a habit of smoking and alcohol consumption. 


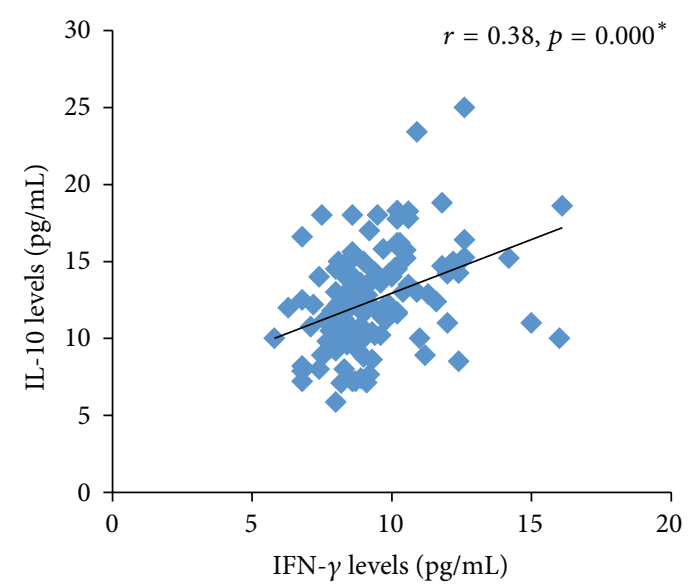

(a)

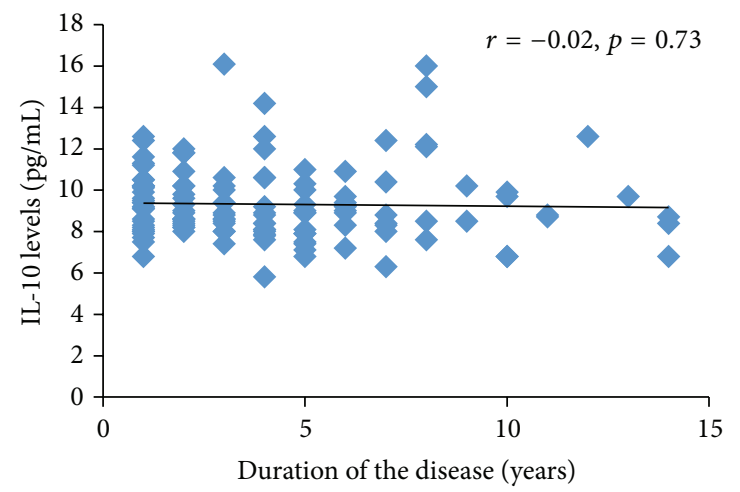

(c)

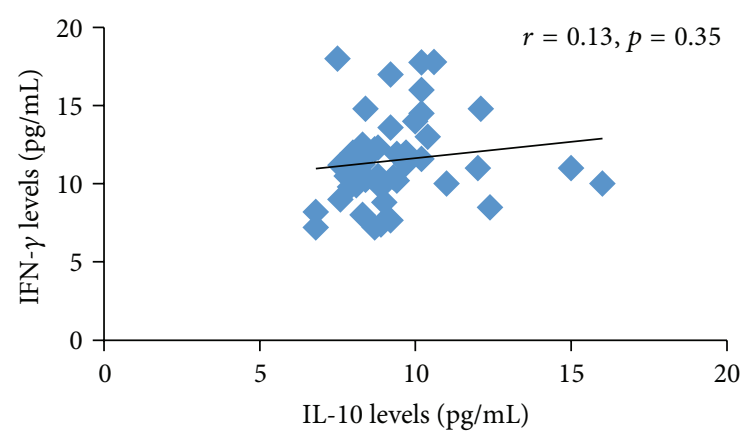

(e)

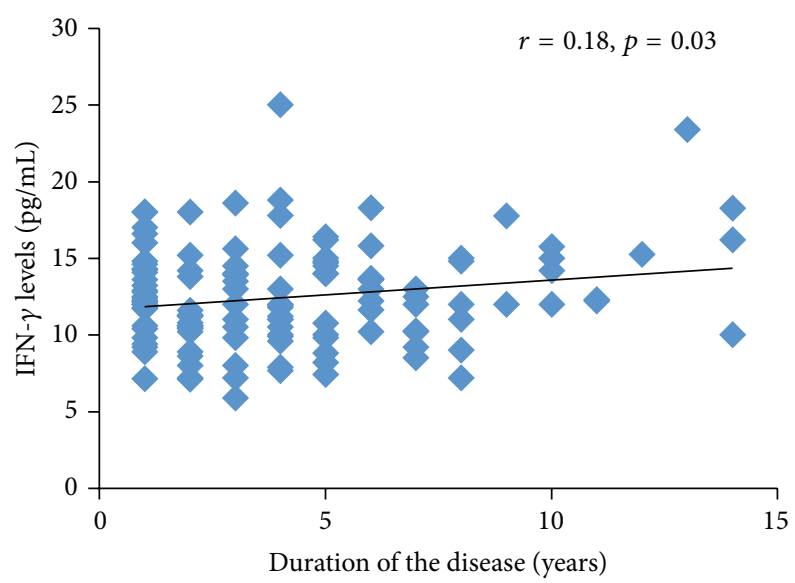

(b)

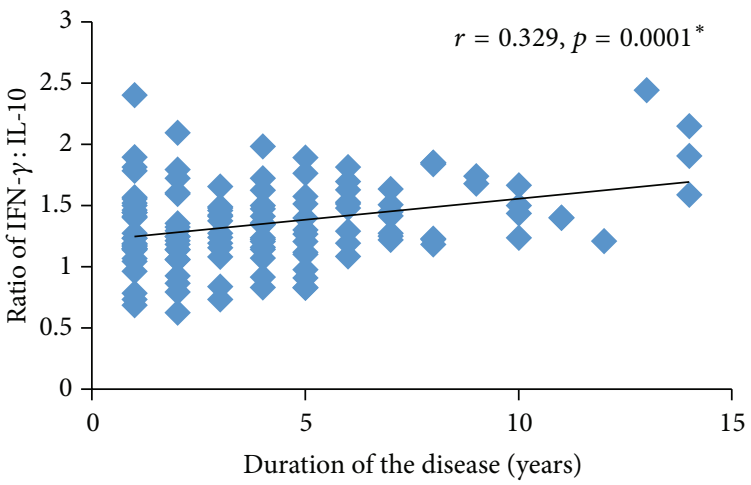

(d)

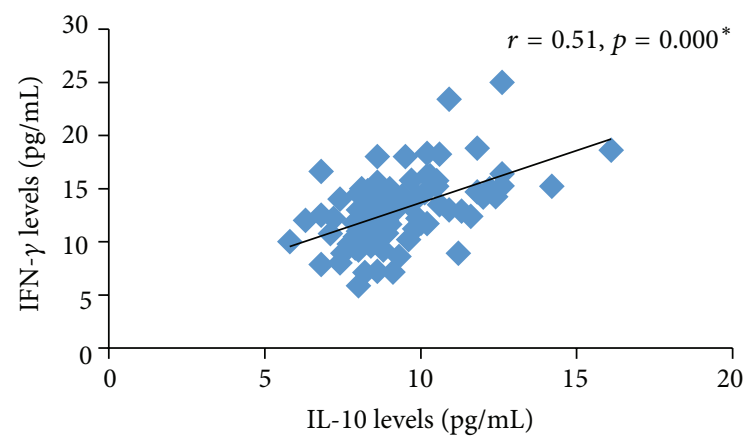

(f)

FIGURE 2: Representing correlation of (a) IL-10 and IFN- $\gamma$ levels in patients, (b) duration of the disease with IFN- $\gamma$ levels, (c) duration of the disease with IL-10 levels, (d) duration of the disease with IFN- $\gamma$ : IL-10 ratio, (e) IL-10 with IFN- $\gamma$ levels in stable vitiligo patients, and (f) IL-10 with IFN- $\gamma$ levels in active vitiligo patients.

increase in IL-10 and IFN- $\gamma$ levels at the individual patient level. Shi and Erf showed a strong association of IFN- $\gamma$ with a parallel increase in IL-10 suggesting the Th1 polarization in Smyth line chickens. The amount of the anti-inflammatory cytokine IL-10 might have increased to be a counterpart of the proinflammatory effect which could be insufficient to control the proinflammatory cascade of events that are responsible for melanocyte destruction [19].

The basis behind various clinical presentations of NSV such as nondermatomal, acrofacial, mucosal, and focal variants at the time of diagnosis is not well understood. In the present study, evaluation of serum IFN- $\gamma$ levels and the ratio of IFN- $\gamma$ : IL-10 among clinical variants of NSV exhibited a significant difference. However, no difference was observed in relation to serum concentrations of IL-10 alone. Acrofacial vitiligo showed the highest IFN- $\gamma$ and the least IL-10 concentrations among the variants. These observations may gain support by the findings of Gupta et al. and Shah et al. who stated that acrofacial and nondermatomal vitiligo conditions are very difficult to treat $[28,29]$, which indirectly indicates the relation between higher proinflammatory environment and clinical presentation. The ratio of IFN- $\gamma$ : IL-10 
may reflect the state of inflammation in NSV pathogenesis. However, in order to support our observation, studies with a large sample size in relation to clinical variants should be taken up.

In addition, the data was evaluated by taking the duration of the disease into consideration, which showed a positive correlation with IFN- $\gamma$ and IFN- $\gamma$ : IL-10 ratio but not with IL-10 levels. It gives a hint that systemic proinflammatory environment may increase with increase in the duration of the disease. Due to lack of literature in this aspect, we claim indirect support from another study that showed higher grades of response to UVB treatment in patients with recent vitiligo compared to those with long-standing disease [30].

Appraisal of proinflammatory and anti-inflammatory cytokines in relation to active and stable vitiligo revealed significantly elevated amounts of IFN- $\gamma$ and IFN- $\gamma$ : IL-10 ratio and no variation in the serum concentrations of IL10. Similar to our observation, another Indian study has also reported higher IFN- $\gamma$ serum concentrations in active vitiligo compared to the stable one [23]. Further, our results are supported by the cell based studies which showed an increased number of $\mathrm{CD}^{+} \mathrm{T}$ cells compared to $\mathrm{CD}^{+} \mathrm{T}$ cells in the blood samples of active vitiligo compared to the stable one. Our observation of elevated IFN- $\gamma$ : IL-10 ratio in the former group compared to the latter also goes hand in hand with the report of Dwivedi et al. showing decreased ratio of $\mathrm{CD}^{+} / \mathrm{CD}^{+}$count [18]. Further, in vitro direct analysis of margins of vitiliginous skin showed polarized type $1 \mathrm{~T}$ cells $\left(\mathrm{CD}^{+}\right.$and particularly $\left.\mathrm{CD} 8^{+}\right)$that predominantly secrete IFN- $\gamma$ and TNF- $\alpha$ cytokines that are associated with the destruction of melanocytes during active vitiligo [31].

Significantly elevated concentrations of IFN- $\gamma$ and the ratio of IFN- $\gamma$ :IL-10 in male patients with social habits suggest smoking and alcohol may contribute to increased inflammatory response. Smoking and alcohol consumption are known to affect the balance between oxidants and antioxidants $[32,33]$.

Six to thirty-eight percent of patients with this complex disorder were reported to be associated with family background of vitiligo [34]. The present study was comprised of $40 \%$ of the patients with the familial history of NSV. Hence, it was felt that the analysis should be carried out taking this aspect into consideration in relation to cytokines. Interestingly, we found that the patients with familial history of NSV exhibited significantly elevated concentrations of IFN- $\gamma$ : IL-10 ratio compared to those without. Further, there was a lack of variation with respect to individual levels of IFN- $\gamma$ and IL-10 between the two subgroups of patients. There are no reports available in the literature in alliance with cytokine levels in vitiligo patients with familial background of vitiligo. We took an initiation to correlate the link between familial history and cytokine levels and their ratio. Based on our present observations, it can be explored towards the potentiality of the ratio as an immunological marker for identifying the high risk individuals from unaffected sibs of NSV patients. In addition, tracing the cosegregation of genetic variants of IFN- $\gamma$ and IL-10 through family studies may provide the role of these cytokines in NSV pathogenesis.
In conclusion, further studies assessing other pro- and anti-inflammatory cytokines and their ratios at systemic as well as epidermal cytokines (lesional and perilesional) before and after the treatment with a large sample size in various ethnic groups are warranted to confirm our results and to open up the new therapeutic options.

\section{Conflict of Interests}

The authors declare no conflict of interests regarding the publication of this paper.

\section{Acknowledgment}

The authors thank the Department of Science and Technology (DST), New Delhi, for providing the financial support no. SR/SO/HS/0151/2010.

\section{References}

[1] R. V. Koranne, D. Derm, and K. G. Sachdeva, "Vitiligo," International Journal of Dermatology, vol. 27, no. 10, pp. 676681, 1988.

[2] B. Esmaeili, S. A. R. Rezaee, P. Layegh et al., "Expression of IL17 and COX2 gene in peripheral blood leukocytes of vitiligo patients," Iranian Journal of Allergy, Asthma and Immunology, vol. 10, no. 2, pp. 81-89, 2011.

[3] J. J. Nordlund and J. P. Ortonne, "Vitiligo vulgaris," in The Pigmentary System: Physiology and Pathophysiology, pp. 513551, Oxford University Press, New York, NY, USA, 1988.

[4] P. Jahan, R. Cheruvu, S. Tippisetty, P. L. Komaravalli, V. Valluri, and M. Ishaq, "Association of FOXP3 (rs3761548) promoter polymorphism with nondermatomal vitiligo: a study from India," Journal of the American Academy of Dermatology, vol. 69, no. 2, pp. 262-266, 2013.

[5] E. M. Shajil, S. Chatterjee, D. Agrawal, T. Bagchi, and R. Begum, "Vitiligo: pathomechanisms and genetic polymorphism of susceptible genes," Indian Journal of Experimental Biology, vol. 44, no. 7, pp. 526-539, 2006.

[6] M. Rashigi, P. Agarwal, J. M. Richmond et al., "CXCL10 is critical for the progression and maintenance of depigmentation in a mouse model of vitiligo," Science Translational Medicine, vol. 6, no. 223, Article ID 223ra23, 2014.

[7] E. H. Kemp, E. A. Waterman, and A. P. Weetman, "Immunological pathomechanisms in vitiligo," Expert Reviews in Molecular Medicine, vol. 3, no. 20, pp. 1-22, 2001.

[8] J. G. van den Boorn, D. Konijnenberg, T. A. M. Dellemijn et al., "Autoimmune destruction of skin melanocytes by perilesional T cells from vitiligo patients," Journal of Investigative Dermatology, vol. 129, no. 9, pp. 2220-2232, 2009.

[9] M. Feldmann, F. M. Brennan, and R. Maini, "Cytokines in autoimmune disorders," International Reviews of Immunology, vol. 17, no. 1-4, pp. 217-228, 1998.

[10] S. Moretti, A. Spallanzani, L. Amato et al., "New insights into the pathogenesis of vitiligo: imbalance of epidermal cytokines at sites of lesions," Pigment Cell Research, vol. 15, no. 2, pp. 8792, 2002.

[11] S. Singh, U. Singh, and S. S. Pandey, "Serum concentration of IL-6, IL-2, TNF- $\alpha$, and IFN $\gamma$ in vitiligo patients," Indian Journal of Dermatology, vol. 57, no. 1, pp. 12-14, 2012. 
[12] A. Alkhateeb, P. R. Fain, A. Thody, D. C. Bennett, and R. A. Spritz, "Epidemiology of vitiligo and associated autoimmune diseases in Caucasian probands and their families," Pigment Cell Research, vol. 16, no. 3, pp. 208-214, 2003.

[13] F. Paolieri, C. Salmaso, M. Battifora et al., "Possible pathogenetic relevance of interleukin- $1 \beta$ in 'destructive' organspecific autoimmune disease (Hashimoto's thyroiditis)," Annals of the New York Academy of Sciences, vol. 876, pp. 221-228, 1999.

[14] K. W. Moore, R. de Waal Malefyt, R. L. Coffman, and A. O'Garra, "Interleukin-10 and the interleukin-10 receptor," Annual Review of Immunology, vol. 19, pp. 683-765, 2001.

[15] D. F. Fiorentino, A. Zlotnik, P. Vieira et al., "IL-10 acts on the antigen presenting cell to inhibit cytokine production by Th1 cells," The Journal of Immunology, vol. 146, no. 10, pp. 3444-3451, 1991.

[16] C. L. Huang, J. J. Nordlund, and R. Boissy, "Vitiligo: a manifestation of apoptosis?" American Journal of Clinical Dermatology, vol. 3, no. 5, pp. 301-308, 2002.

[17] M. K. Tembhre, V. K. Sharma, A. Sharma, P. Chattopadhyay, and S. Gupta, "T helper and regulatory T cell cytokine profile in active, stable and narrow band ultraviolet $\mathrm{B}$ treated generalized vitiligo," Clinica Chimica Acta, vol. 424, pp. 27-32, 2013.

[18] M. Dwivedi, N. C. Laddha, P. Arora, Y. S. Marfatia, and R. Begum, "Decreased regulatory $\mathrm{T}$ cells and $\mathrm{CD} 4^{+} / \mathrm{CD} 8^{+}$ratio correlate with disease onset and progression in patients with generalized vitiligo," Pigment Cell \& Melanoma Research, vol. 26, no. 4, pp. 586-591, 2013

[19] F. Shi and G. F. Erf, "IFN- $\gamma$, IL-21, and IL-10 co-expression in evolving autoimmune vitiligo lesions of smyth line chickens," Journal of Investigative Dermatology, vol. 132, no. 3, pp. 642-649, 2012.

[20] J. E. Harris, T. H. Harris, W. Weninger, E. J. Wherry, C. A. Hunter, and L. A. Turka, "A mouse model of vitiligo with focused epidermal depigmentation requires IFN- $\gamma$ for autoreactive $\mathrm{CD}^{+}$T-cell accumulation in the skin," Journal of Investigative Dermatology, vol. 132, no. 7, pp. 1869-1876, 2012.

[21] P. K. Nigam, P. K. Patra, P. K. Khodiar, and J. Gual, "A study of blood CD3+, CD4+, and CD8+ T cell levels and CD4+:CD8+ ratio in vitiligo patients," Indian Journal of Dermatology, Venereology and Leprology, vol. 77, no. 1, p. 111, 2011.

[22] R. K. Gregg, L. Nichols, Y. Chen, B. Lu, and V. H. Engelhard, "Mechanisms of spatial and temporal development of autoimmune vitiligo in tyrosinase-specific tcr transgenic mice," The Journal of Immunology, vol. 184, no. 4, pp. 1909-1917, 2010.

[23] M. Dwivedi, N. C. Laddha, K. Shah, B. J. Shah, and R. Begum, "Involvement of interferon-gamma genetic variants and intercellular adhesion molecule-1 in onset and progression of generalized Vitiligo," Journal of Interferon and Cytokine Research, vol. 33, no. 11, pp. 646-659, 2013.

[24] J. J. Yohn, M. M. Critelli, B. Lyons, and D. A. Norris, "Modulation of melanocyte intercellular adhesion molecule-1 by immune cytokines," Journal of Investigative Dermatology, vol. 95, no. 2, pp. 233-237, 1990.

[25] Z. A. Taher, G. Lauzon, S. Maguiness, and M. T. Dytoc, "Analysis of interleukin-10 levels in lesions of vitiligo following treatment with topical tacrolimus," British Journal of Dermatology, vol. 161, no. 3, pp. 654-659, 2009.

[26] P. E. Grimes, R. Morris, E. Avaniss-Aghajani, T. Soriano, M. Meraz, and A. Metzger, "Topical tacrolimus therapy for vitiligo: therapeutic responses and skin messenger RNA expression of proinflammatory cytokines," Journal of the American Academy of Dermatology, vol. 51, no. 1, pp. 52-61, 2004.
[27] R. Colucci, F. Dragoni, and S. Moretti, "Oxidative stress and immune system in vitiligo and thyroid diseases," Oxidative Medicine and Cellular Longevity, vol. 2015, Article ID 631927, 7 pages, 2015.

[28] S. Gupta, T. Narang, M. J. Olsson, and J. P. Ortonne, "Surgical management of vitiligo and other leukodermas: evidence-based practice guidelines," in Surgical Management of Vitiligo, S. Gupta, M. J. Olsson, A. J. Kanwar, and J.-P. Ortonne, Eds., chapter 9, Blackwell Publishing, Oxford, UK, 2007.

[29] B. H. Shah, S. P. Joshipura, and J. K. Thakkar, "Surgical treatment in acrofacial vitiligo," Indian Journal of Dermatology, Venereology and Leprology, vol. 60, no. 1, pp. 26-27, 1994.

[30] Z. Hallaji, M. Ghiasi, A. Eisazadeh, and M. Rayati Damavandi, "Evaluation of the effect of disease duration in generalized vitiligo on its clinical response to narrowband ultraviolet $\mathrm{B}$ phototherapy," Photodermatology Photoimmunology and Photomedicine, vol. 28, no. 3, pp. 115-119, 2012.

[31] A. Wańkowicz-Kalińska, R. M. J. G. J. van den Wijngaard, B. J. Tigges et al., "Immunopolarization of $\mathrm{CD} 4^{+}$and $\mathrm{CD} 8^{+} \mathrm{T}$ cells to type-1-like is associated with melanocyte loss in human vitiligo," Laboratory Investigation, vol. 83, no. 5, pp. 683-695, 2003.

[32] B. Isik, A. Ceylan, and R. Isik, "Oxidative stress in smokers and non-smokers," Inhalation Toxicology, vol. 19, no. 9, pp. 767-769, 2007.

[33] J. W. Shin, K. M. Nam, H. R. Choi et al., "Erythrocyte malondialdehyde and glutathione levels in vitiligo patients," Annals of Dermatology, vol. 22, no. 3, pp. 279-283, 2010.

[34] J. P. Ortonne, D. B. Mosher, and T. B. Fitzpatrick, "Vitiligo and other hypomelanoses of hair and skin," in Topics in Dermatology, J. P. Ortonne, D. B. Mosher, and T. B. Fitzpatrick, Eds., pp. 257-258, Plenum Medical Book, New York, NY, USA, 1983. 


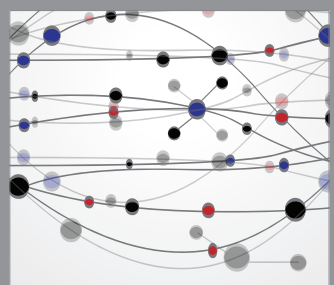

The Scientific World Journal
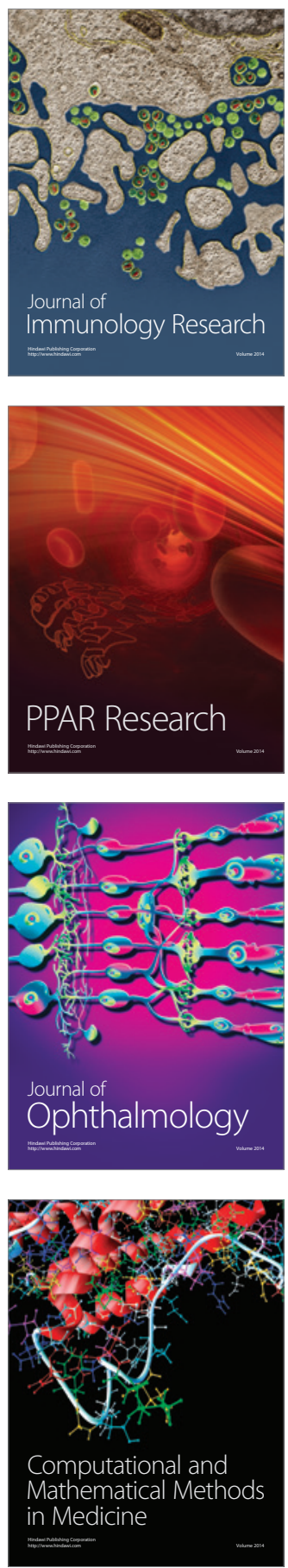

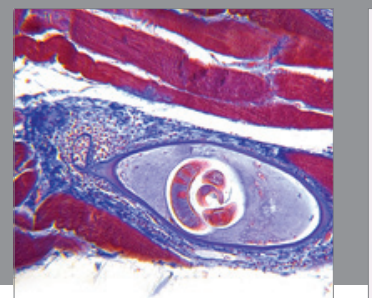

Gastroenterology

Research and Practice
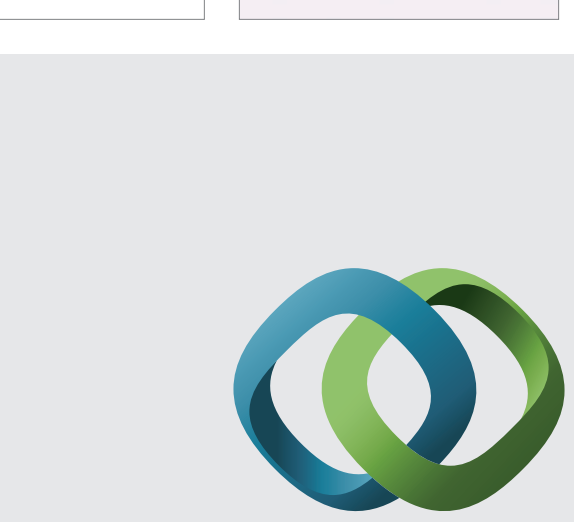

\section{Hindawi}

Submit your manuscripts at

http://www.hindawi.com
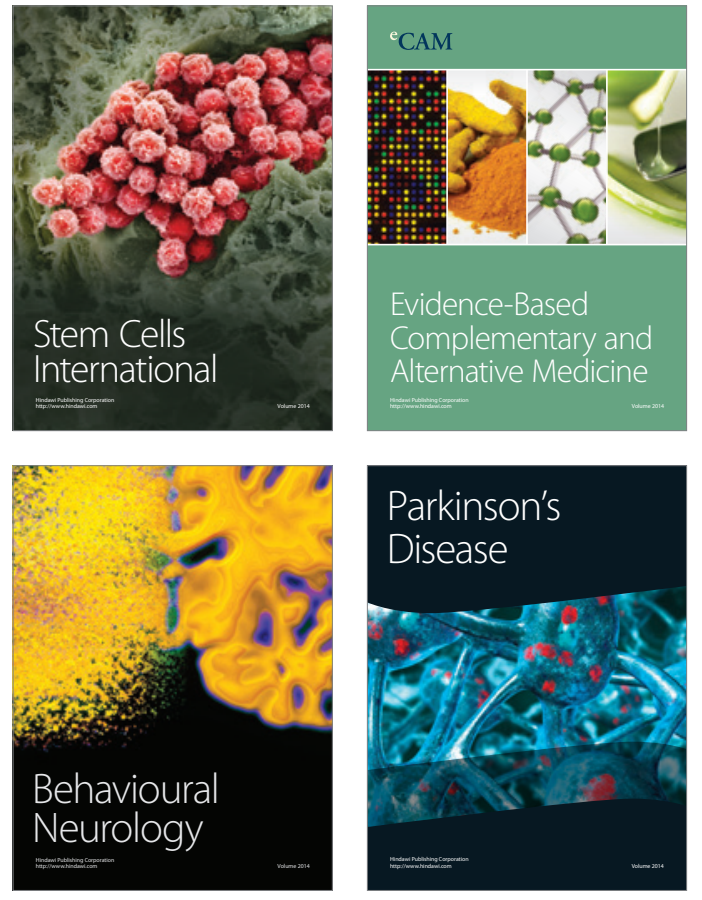
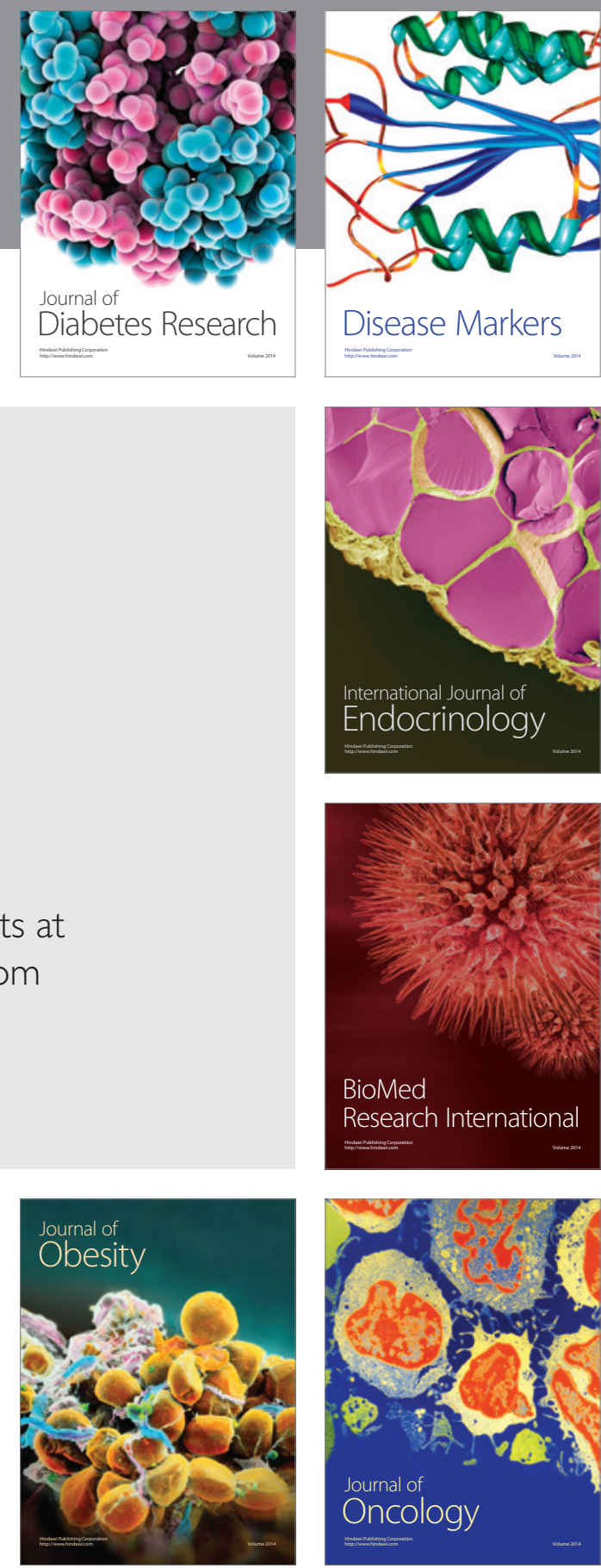

Disease Markers
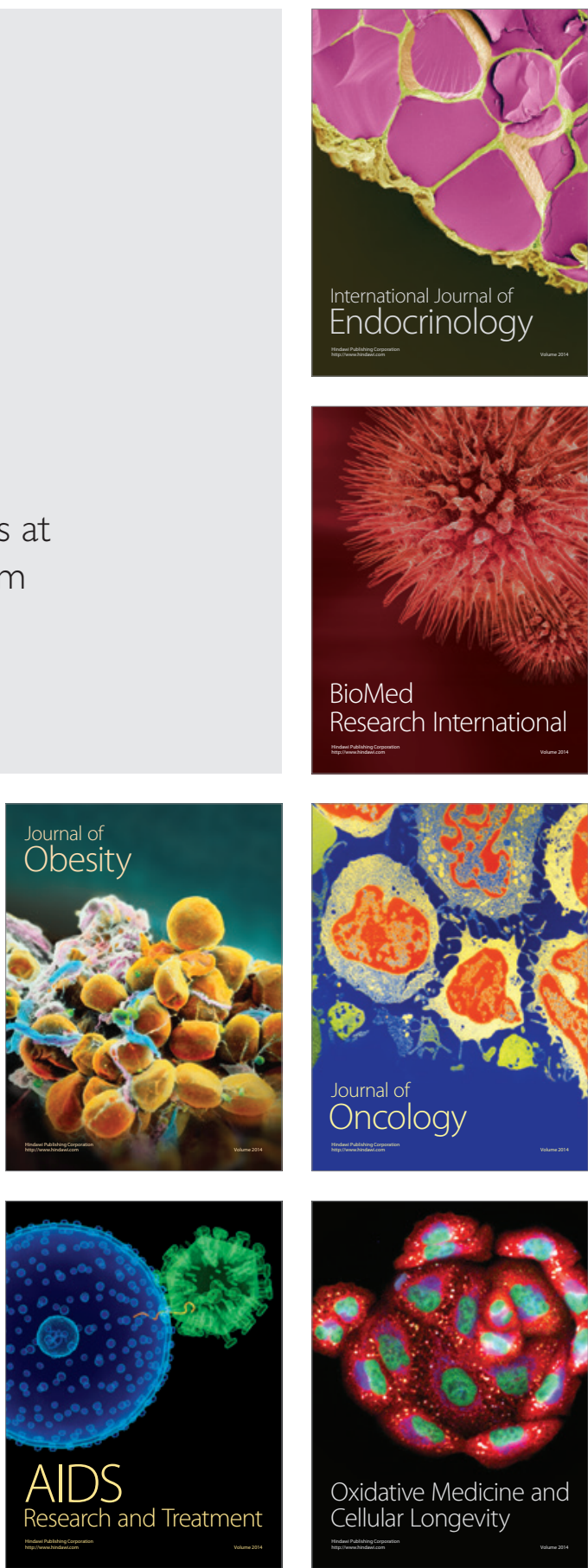